\title{
Part of plasmapheresis with plasma filtration adsorption combined with continuous hemodiafiltration in the treatment of severe acute liver failure
}

\author{
MAOQIN LI, ZHIDONG WANG, YINING WANG, CHANGHONG DU, SONGHAI LI, \\ ZAIXIANG SHI and BO LU
}

Department of Intensive Care Unit, Xuzhou Central Hospital, Xuzhou, Jiangsu 221009, P.R. China

Received February 8, 2016; Accepted July 4, 2016

DOI: $10.3892 / \mathrm{etm} .2016 .3633$

\begin{abstract}
The present study is a retrospective analysis of 11 cases with severe acute liver failure combined with multiple organ dysfunction syndrome (MODS) performed during the period June, 2012 to December, 2014. After part of plasmapheresis with plasma filtration adsorption combined with continuous hemodiafiltration treatment, good curative effects were obtained and the main clinical symptoms and biochemical index were significantly improved. Following treatment, 8 of the 11 patients survived at a survival rate of $72.7 \%$, and 3 patients succumbed with a mortality of $27.3 \%$. The results suggested that part of plasmapheresis with plasma filtration adsorption combined with continuous venovenous hemodiafiltration (CVVHDF) treatment is beneficial in the removal of metabolites and toxins. Additonally, it can effectively improve liver function and clinical symptoms, improve hepatic encephalopathy, correct the disorder of internal environment, and improve the prognosis of patients.
\end{abstract}

\section{Introduction}

Acute liver failure (ALF) can be caused by viral and non-viral hepatitis, often accompanied by serious metabolic disorder and toxic substance accumulation. Continuous sustained release as well as the accumulation of a large number of endogenous toxins and inflammatory medium can promote liver injury and inhibit the regeneration of liver cells, resulting in a vicious cycle (1). In spite of the execution of many medical treatments for liver protection, such as the treatment of jaundice, the mortality rate remains at over $70 \%$ (2).

Artificial liver support system (ALSS) can replace liver function temporarily and partially remove harmful

Correspondence to: Dr Maoqin Li, Department of Intensive Care Unit, Xuzhou Central Hospital, Xuzhou, Jiangsu 221009, P.R. China E-mail: limaoqinxi@126.com

Key words: acute liver failure, severe, part of plasmapheresis, plasma filtration adsorption, continuous hemodiafiltration substances, supply biological active substances, create good internal environment and gain valuable time for cell regeneration and functional recovery in patients (1). For patients with severe acute liver failure, the combination therapy of blood purification technologies constitutes a research hotpot of non-biological artificial liver treatments.

In the present study, part of plasmapheresis with plasma filtration adsorption combined with continuous hemodiafiltration was used in patients with severe acute liver failure, which achieved good treatment effect.

\section{Patients and methods}

General data. Eleven cases, including 5 men and 6 women, aged 35.3 \pm 9.95 years (range, 24-76 years) with severe acute liver failure combined with multiple organ dysfunction syndrome (MODS) with a hepatic MODS score of grade IV (total bilirubin $>240 \mu \mathrm{mol} / \mathrm{l}$ ), were admitted to the Department of Intensive Care Unit of the Xuzhou Central Hospital between June, 2012 and December, 2014. Acute physiology and chronic health evaluation II (APACHE II) was $4.3 \pm 5.4$ points, and sequential organ failure assessment (SOFA) score was 12.03 \pm 3.14 . The protopathy included 2 cases with drug toxicity, 5 cases with pregnancy fatty liver, 1 patient with severe trauma, 1 patient with severe infection, 1 patient with gradual and acute liver failure and 1 patient with unknown reason.

The study was approved by the Ethics Committee of Xuzhou Central Hospital. Written informed consent was obtained from the patients or family.

Treatment methods. In addition to conventional medicine and symptomatic and supportive treatment, each patient was treated with part of plasmapheresis with plasma filtration adsorption combined with continuous venovenous hemodiafiltration (CVVHDF). Following the creation of temporary access by cannula of femoral vein or internal jugular vein, 11 patients received plasmapheresis at bedside using a plasma separator (Gambro PF 2000N, Zurich, Switzerland). In addition, fresh plasma of $1,500 \mathrm{ml}$ was replaced each time, with a blood flow velocity of $80-120 \mathrm{ml} / \mathrm{min}$, plasma separation speed of $25-30 \mathrm{ml} / \mathrm{min}$, and a replacement time of approximately $2 \mathrm{~h}$. Following completion of the single 
plasmapheresis, the original plasma separator was employed to proceed with the resin adsorption of plasma separation or bilirubin adsorption. Two GAMBRO-BMM10-1K (Flensburg, Germany) single pump machines were used to conduct plasma perfusion, and neuter macroporous adsorption resin (HA330-II, a blood plasma perfusion) using perfusion apparatus. When total bilirubin was $\geq 350 \mu \mathrm{mol} / 1$, perfusion apparatus was changed by ion exchange resin (BS330 bilirubin adsorption column) (both from Jafron Biomedical Co., Ltd., Zhuhai, China). Pre-filled vein indwelling catheter connected with the arterial canal of plasma shunt and blood pump was used to drain blood, with gradual adjustment for the blood pump flow velocity of $80-150 \mathrm{ml} / \mathrm{min}$ and plasma pump flow velocity of $25-50 \mathrm{ml} / \mathrm{min}$. Arteries and venous pressure of the two blood perfusion (BP) apparatuses were monitored, and when the adsorption ability of plasma perfusion achieved saturation, the perfusion machine was removed, and the time of plasma perfusion treatment was 3-5 h. Following plasma filtration adsorption, GAMBRO-FLUX or GAMBROPRISMA hemofiltration apparatus was used for continuous hemodialysis and filtration with CVVHDF treatment for 12-24 h. Heparin-free or heparin-limited method was used based on the blood coagulation function of patients. This combined technology treatment was repeated twice (once to three times) on average. Thus, all 11 patients received a total of 23 sessions of therapies.

During the process of treatment, consciousness, heart rate (HR), mean arterial pressure (MAP), and percutaneous oxygen saturation were monitored constantly. Prior to and following each treatment, monitor liver and kidney function indexes, arterial blood gas $\left(\mathrm{PH}, \mathrm{PaO}_{2}\right.$ and $\left.\mathrm{PaCO}_{2}\right)$, blood routine, electrolytes, and blood coagulation function were also checked.

Statistical analysis. Data were presented as mean \pm standard deviation. Statistical analysis was performed using software SPSS 12.0 (Chicago, IL, USA). Comparisons prior and subsequent to treatment were applied using the t-test. $\mathrm{P}<0.05$ was considered statistically significant.

\section{Results}

Clinical curative effect. After part of plasmapheresis with plasma filtration adsorption combined with CVVHDF treatment was applied to the 11 patients, their health improved significantly, with manifestation of gradual improvement of irritability, logomania, haziness of spirit-affect and coma, clear reduction of jaundice, gradual improvement of bleeding tendency, gradual stability of vital signs, increase of urine and release of edema. Of the 11 patients, 8 patients survived with a survival rate of $72.7 \%(8 / 11)$, and 3 patients succumbed to the disease, with a mortality of $27.3 \%(3 / 11)$.

Comparison of the two methods prior to and after treatment. After plasmapheresis with plasma filtration adsorption combined with CVVHDF treatment, the level of TBIL, DBIL, ALT, AST, PT, BUN and PT decreased significantly, and ALB, and PLT increased, compared with those prior to treatment. The difference was significant $(\mathrm{P}<0.05)$. The change of platelet was not obvious $(\mathrm{P}>0.05)$ (Table I).
Table I. Comparison of biochemical indexes with three artificial liver technique combination prior to and after treatment (mean $\pm \mathrm{SD})$.

\begin{tabular}{lccl}
\hline $\begin{array}{l}\text { Biochemical } \\
\text { indexes }\end{array}$ & $\begin{array}{c}\text { Before } \\
\text { treatment }\end{array}$ & $\begin{array}{c}\text { After } \\
\text { treatment }\end{array}$ & P-value \\
\hline TBIL $(\mu \mathrm{mol} / \mathrm{l})$ & $418.18 \pm 188.92$ & $257.44 \pm 151.42$ & $<0.001$ \\
$\operatorname{DBIL~}(\mu \mathrm{mol} / \mathrm{l})$ & $277.32 \pm 245.93$ & $108.35 \pm 61.11$ & $<0.01$ \\
$\operatorname{ALT}(\mu / \mathrm{l})$ & $498.74 \pm 168.66$ & $161.02 \pm 68.92$ & $<0.001$ \\
$\operatorname{AST}(\mu / \mathrm{l})$ & $377.32 \pm 245.93$ & $108.35 \pm 61.11$ & $<0.001$ \\
$\operatorname{PT~}(\mathrm{S})$ & $25.65 \pm 15.74$ & $18.70 \pm 15.03$ & $<0.05$ \\
$\operatorname{ALB}(\mathrm{g} / \mathrm{l})$ & $27.31 \pm 4.55$ & $30.33 \pm 4.25$ & $<0.05$ \\
$\operatorname{PLT}\left(\mathrm{x} 10^{9} / \mathrm{l}\right)$ & $80.89 \pm 51.21$ & $78.31 \pm 46.65$ & $<0.05$ \\
\hline
\end{tabular}

\section{Discussion}

Acute liver failure occurs suddenly and its development is rapid leading to poor prognosis, and therefore, more attention should be paid to its occurrence (3). Acute liver failure is a MODS mainly with acute liver failure. The effect of simple conservative treatment is not good, while blood purification technology can replace the metabolism function of liver and support multiple organ function in patients effectively at the same time (4). Liver failure treated by artificial liver plays a crucial role in the decrease of serum bilirubin of patients, clears or reduces the accumulation of toxic substances in the body, and improves internal environment. Sentürk et al used fractionated plasma separation and the adsorption system to treat acute liver failure, and in that clinical study, the survival rate of patients was $48.1 \%$ (5). He et al applied coupled plasma filtration adsorption with hemofiltration in the treatment of patients with MODS accompanied with acute liver failure in ICU, and the survival rate was $45.5 \%$ (6). Liu et al applied ALSS by plasmapheresis and CVVHDF to treat acute liver failure combined with MODS, and the survival rate was $42.5 \%$ (7). Yin et al applied DPMAS to treat 42 cases of liver failure, and the survival rate was $64.29 \%$ (8). The patients' livers in this group were above grade IV according to the MODS score with PTA $\leq 30 \%$, belonging to middle-late stage of liver failure in terms of guideline stages of liver failure (9). A combination of three types of non-biological artificial liver treatments was made and of the 11 patients, 8 patients survived with a survival rate of $72.7 \%$, a survival rate that was significantly improved as compared to the abovementioned studies. The increased survival rate observed in our study, may be associated with the main causes that lead to acute liver failure, pregnancy fatty liver, drug poisoning, trauma and infection. For this type of non-viral acute liver failure, if the pathogenesis is removed, the prognosis of patients may be improved with via the effective combination of artificial liver support therapy.

Plasma exchange (PE) can remove endogenous toxins such as endotoxin, bilirubin, and bile acid. Macromolecular material combined with plasma proteins, and circulating immune complex in patients with liver failure can, at the same time, complement blood coagulation factors and improve coagulation function (10). Supplementation of plasma albumin, 
opsonin, immunoglobulin and other bioactive substances results in the affirmation of the PE curative effect (10). However, the treatment needs a large quantity of plasma and there is a possible occurrence of transfusion transmitted infection. Additionally, $\mathrm{PE}$ is capable of removing material that promote the growth of liver cells, which may affect liver regeneration and the long-term curative effect. Part PE method was employed in the present study; however, due to a blood shortage, a small dose of PE can reduce the dose of plasma, while part PE combined with plasma adsorption constitutes an effective treatment. By contrast, the increased cost of adsorption is equal to the reduction in the cost of plasma dose; thus, the medical expense of the patient does not increase. Furthermore, BP adsorbs cell toxic substances in blood, aromatic amino acids, phenol, indole, and short chain fatty acids mainly in patients with hepatic failure. The HA-type resin perfusion machine used in BP belongs to macroporous resin, with the adsorption substance at a molecular weight of 500-5,000 kDa, which can adsorb many toxins that combine with protein and cell toxic substances that inhibit liver cell regeneration (11). The bilirubin adsorption column increased the capacity of bilirubin significantly (12). CVVHDF can clear medium molecular substance, blood ammonia and other toxic substances, such as false neurotransmitters, free fatty acids, mercaptan, and aromatic amino acids in patients with acute liver failure, increase the content of CAMP in cerebrospinal fluid, improve the energy metabolism in brain, and relieve and improve hepatic encephalopathy (13). At the same time, it can control capacity accurately, eliminate solute and liquid slowly and continuously, adjust the acid-base balance of water electrolytes and reduce the occurrence of brain edema due to acute liver failure. The results of the present study suggest that plasmapheresis with plasma filtration adsorption combined with CVVHDF treatment technology is more beneficial to remove metabolites and toxins and maintain the stability of internal environment.

Our experience suggests that there are many different modes of artificial liver treatment in that we can select non-biological artificial liver treatment of different combinations according to the patients' conditions. Part plasmapheresis with HA330-II plasma perfusion (or BS bilirubin plasma adsorption) combined with CVVHDF can absorb bilirubin and remove many related toxic substances of liver failure such as inflammatory mediators (14). Thus, it can effectively improve liver function and the related clinical symptoms, improve hepatic encephalopathy and correct internal environment disturbances, as well as improve short-term efficacy and the survival rate. It can save plasma resources and reduce the rebound phenomenon of simplex exchange (14). This type of combination of treatment deserves to be popularized in the clinical treatment of acute hepatic failure. These three types of combination of artificial liver treatment technologies are easier to operate with better clinical curative effect, compared to plasma separation adsorption combined with CVVHDF technology. However, the small number of cases in the present study constitute a limitation and further investigations should be conducted.

In terms of safety, the patients in the present study underwent PE with resin absorption combined with CVVHDF therapy. During this process, high and low blood pressure complications did not occur. The number of white-blood cells and platelets were not altered. The therapy was tolerated in the course of the treatment, and there were no complications and artificial liver was interrupted for only one case.

In conclusion, part of plasmapheresis with plasma filtration adsorption combined with CVVHDF treatment is beneficial to remove metabolites and toxins. In addition, it can effectively improve the liver function and clinical symptoms, improve hepatic encephalopathy, correct the disorder of internal environment, and improve the prognosis of patients.

\section{Acknowledgements}

The present study was funded by the Funding Project of Medical Leading Talents in Xuzhou (grant no. 201109).

\section{References}

1. Li LJ, Zhang YM, Liu XL, Du WB, Huang JR, Yang Q, Xu XW and Chen YM: Artificial liver support system in China: A review over the last 30 years. Ther Apher Dial 10: 160-167, 2006

2. Nevens F and Laleman W: Artificial liver support devices as treatment option for liver failure. Best Pract Res Clin Gastroenterol 26: 17-26, 2012.

3. Lee WM, Squires RJ, Nyberg SL, Doo E and Hoofnagle JH: Acute liver failure: Summary of a workshop. Hepatology 47: 1401-1415, 2008

4. Freeman JG, Matthewson K and Record CO: Plasmapheresis in acute liver failure. Int J Artif Organs 9: 433-438, 1986.

5. Sentürk E, Esen F, Ozcan PE, Rifai K, Pinarbași B, Cakar N and Telci L: The treatment of acute liver failure with fractionated plasma separation and adsorption system: experience in 85 applications. J Clin Apher 25: 195-201, 2010.

6. He CS, Shi W, Ye ZM, Liang XL, Zhang B, Liu SX and Qin TH: Experiment and clinical research of coupled plasma filtration adsorption with hemofiltration on the treatment of multiple organ dysfunction syndrome. Chin Crit Care Med 19: 47-49, 2007 (In Chinese).

7. Liu Y, He JQ, Che DP and Yang WL: Treatment of artificial liver support system on acute liver failure combined with multiple organ dysfunction syndyrome. Chin Crit Care Med 18: 431-432, 2006 (In Chinese).

8. Yin G, Ya C, Li Q, et al: Clinical research of DPMAS treatment on liver failure by the combination of two blood perfusion machines. Chin J Crit Care Med 25: 738-742, 2013 (In Chinese).

9. Liver Failure and Artificial Liver Group, Chinese Society of Infectious Diseases, Chinese Medical Association, Severe Liver Diseases and Artificial Liver Group, Chinese Society of Hepatology, Chinese Medical Association: [Diagnostic and treatment guidelines for liver failure]. Zhonghua Gan Zang Bing Za Zhi 14: 643-646, 2006.

10. Morgan SM and Zantek ND: Therapeutic plasma exchange for fulminant hepatic failure secondary to Wilson's disease. J Clin Apher 27: 282-286, 2012.

11. Weston MJ: Charcoal hemoperfusion for liver failure. Lancet 2: 983, 1982

12. Chen J, Han W, Su R, Chen J, Zong W, Wang Y, Wang W, Cheng G, Ou L and Yu Y: Non-ionic macroporous polystyrene adsorbents for removal of serum toxins in liver failure by hemoperfusion. Artif Cells Nanomed Biotechnol: Feb 2, 2016 (Epub ahead of print).

13. Ryska O, Pantoflicek T, Laszikova E, Prazak J, Koblihova E and Ryska M: Artificial liver support system reduces intracranial pressure more effectively than bioartificial system: An experimental study. Int J Artif Organs 35: 503-510, 2012.

14. Stutchfield BM, Simpson K and Wigmore SJ: Systematic review and meta-analysis of survival following extracorporeal liver support. Br J Surg 98: 623-631, 2011. 